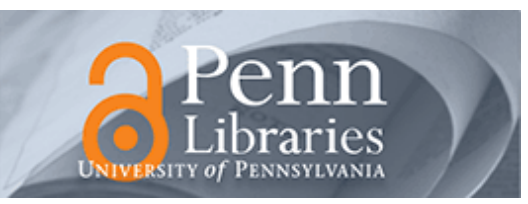

University of Pennsylvania

ScholarlyCommons

February 2001

\title{
Approximate Orientation Steerability Based on Angular Gaussians
}

\author{
Weichan Yu \\ Christian Albrechts University \\ Kostas Daniilidis \\ University of Pennsylvania, kostas@cis.upenn.edu \\ Gerald Sommer \\ Christian Albrechts University
}

Follow this and additional works at: https://repository.upenn.edu/cis_papers

\section{Recommended Citation}

Weichan Yu, Kostas Daniilidis, and Gerald Sommer, "Approximate Orientation Steerability Based on Angular Gaussians", . February 2001.

Copyright 2001 IEEE. Reprinted from IEEE Transactions on Image Processing, Volume 10, Issue 2, February 2001, pages 193-201.

Publisher URL: http://ieeexplore.ieee.org/xpl/tocresult.jsp?isNumber=19508\&puNumber=83

This material is posted here with permission of the IEEE. Such permission of the IEEE does not in any way imply IEEE endorsement of any of the University of Pennsylvania's products or services. Internal or personal use of this material is permitted. However, permission to reprint/republish this material for advertising or promotional purposes or for creating new collective works for resale or redistribution must be obtained from the IEEE by writing to pubs-permissions@ieee.org. By choosing to view this document, you agree to all provisions of the copyright laws protecting it.

This paper is posted at ScholarlyCommons. https://repository.upenn.edu/cis_papers/24

For more information, please contact repository@pobox.upenn.edu. 


\title{
Approximate Orientation Steerability Based on Angular Gaussians
}

\begin{abstract}
Junctions are significant features in images with intensity variation that exhibits multiple orientations. This makes the detection and characterization of junctions a challenging problem. The characterization of junctions would ideally be given by the response of a filter at every orientation. This can be achieved by the principle of steerability that enables the decomposition of a filter into a linear combination of basis functions. However, current steerability approaches suffer from the consequences of the uncertainty principle: In order to achieve high resolution in orientation they need a large number of basis filters increasing, thus, the computational complexity. Furthermore, these functions have usually a wide support which only accentuates the computational burden.

In this paper we propose a novel alternative to current steerability approaches. It is based on utilizing a set of polar separable filters with small support to sample orientation information. The orientation signature is then obtained by interpolating orientation samples using Gaussian functions with small support. Compared with current steerability techniques our approach achieves a higher orientation resolution with a lower complexity. In addition, we build a polar pyramid to characterize junctions of arbitrary inherent orientation scales.

\section{Keywords}

Low-level vision, orientation analysis, steerable filters

\section{Comments}

Copyright 2001 IEEE. Reprinted from IEEE Transactions on Image Processing, Volume 10, Issue 2, February 2001, pages 193-201.

Publisher URL: http://ieeexplore.ieee.org/xpl/tocresult.jsp?isNumber=19508\&puNumber=83

This material is posted here with permission of the IEEE. Such permission of the IEEE does not in any way imply IEEE endorsement of any of the University of Pennsylvania's products or services. Internal or personal use of this material is permitted. However, permission to reprint/republish this material for advertising or promotional purposes or for creating new collective works for resale or redistribution must be obtained from the IEEE by writing to pubs-permissions@ieee.org. By choosing to view this document, you agree to all provisions of the copyright laws protecting it.
\end{abstract}




\title{
Approximate Orientation Steerability Based on Angular Gaussians
}

\author{
Weichan Yu, Kostas Daniilidis, and Gerald Sommer
}

\begin{abstract}
Junctions are significant features in images with intensity variation that exhibits multiple orientations. This makes the detection and characterization of junctions a challenging problem. The characterization of junctions would ideally be given by the response of a filter at every orientation. This can be achieved by the principle of steerability that enables the decomposition of a filter into a linear combination of basis functions. However, current steerability approaches suffer from the consequences of the uncertainty principle: In order to achieve high resolution in orientation they need a large number of basis filters increasing, thus, the computational complexity. Furthermore, these functions have usually a wide support which only accentuates the computational burden.

In this paper we propose a novel alternative to current steerability approaches. It is based on utilizing a set of polar separable filters with small support to sample orientation information. The orientation signature is then obtained by interpolating orientation samples using Gaussian functions with small support. Compared with current steerability techniques our approach achieves a higher orientation resolution with a lower complexity. In addition, we build a polar pyramid to characterize junctions of arbitrary inherent orientation scales.
\end{abstract}

Index Terms-Low-level vision, orientation analysis, steerable filters.

\section{INTRODUCTION}

$\mathbf{J}$ UNCTIONS of gray-value lines or edges in images carry important information for many image processing tasks like point matching in object recognition, point tracking in motion analysis, attentive coding, and line-drawing interpolation [14]. In order to use junctions for such tasks we must be able to localize their corresponding keypoints which are defined as intersection points of lines or edges. Then, we must characterize junctions by means of signatures and classify them in junction categories. Regarding keypoint detection and localization the reader is referred to Foerstner's study [8] and the work of Parida et al. [16] and to the comparison of different operators by Rohr [21], [22]. In this paper we address the problem of junction

Manuscript received May 20, 1999; revised October 13, 2000. W. Yu was supported by DAAD (German Academic Exchange Service). K. Daniilidis and G. Sommer were supported by DFG Grant 320/1-3. K. Daniilidis was also supported by the NSF under Grants CDS-97-03220 and ARO/MURI DAAH04-96-1-0007, Advanced Network and Services, and Penn Research Foundation. The associate editor coordinating the review of this manuscript and approving it for publication was Dr. Eric L. Miller.

W. Yu and G. Sommer are with the Institute of Computer Science, Christian Albrechts University, D-24105 Kiel, Germany (e-mail: wy@ks.informatik.unikiel.de; gs@ks.informatik.uni-kiel.de).

K. Daniilidis is with the GRASP Lab, University of Pennsylvania, Philadelphia, PA 19104-6228 USA (e-mail: kostas@grasp.cis.upenn.edu).

Publisher Item Identifier S 1057-7149(01)00912-5. characterization. The resulting signature can be used for further junction classification.

Junctions are local structures with multiple intrinsic orientations and spatial scales [2]. For the purpose of characterization we project them onto the orientation space and build a one-dimensional (1-D) signature function of the orientation parameter. Such signatures are often obtained by applying a set of filters at different orientations. This leads to an enormous computational load. For example, in order to extract orientation information of a junction, with conventional filter methods we have to rotate the same filter around the keypoint repeatedly. For an angular field of $360^{\circ}$ and a sampling interval of $5^{\circ}$ already 72 rotated copies of the original filter should be applied. The concept of steerability has been introduced in order to reduce this explosion of computational complexity. Steerable filters obtain an analytic model of deformations for further analysis of the grey-value structure [3], [6], [9], [10], [18], [24], [26].

Denoting with $\alpha(\alpha \in \mathbb{R})$ the deformation parameter we define a filter $F(\vec{x})$ with $\vec{x} \in \mathbb{R}^{n}$ as a steerable filter if its deformed versions $F_{\alpha}(\vec{x})$ can be expressed as [13]

$$
F_{\alpha}(\vec{x})=\sum_{k=1}^{N} b_{k}(\alpha) A_{k}(\vec{x})
$$

where $A_{k}(\vec{x})$ and $b_{k}(\alpha)$ are referred to as basis filters and interpolation functions, respectively. The signature $s(\alpha)$ of a junction can be obtained by applying such a steerable filter on the image $I(\vec{x})$

$$
s(\alpha) \stackrel{\text { def }}{=}\left\langle F_{\alpha}(\vec{x}) \mid I(\vec{x})\right\rangle=\sum_{k=1}^{N} \gamma_{k} b_{k}(\alpha)
$$

with

$$
\gamma_{k} \stackrel{\text { def }}{=}\left\langle A_{k}(\vec{x}) \mid I(\vec{x})\right\rangle .
$$

Here, $\langle\cdot \mid \cdot\rangle$ denotes the usual inner product for two real functions $F(\vec{x})$ and $G(\vec{x})$

$$
\langle F(\vec{x}) \mid G(\vec{x})\rangle \stackrel{\text { def }}{=} \int_{\vec{x}} F(\vec{x}) G(\vec{x}) d \vec{x} .
$$

We see the motivation of steerability clearly in (2): The responses of the filter $F_{\alpha}(\vec{x})$ with $\alpha \in \mathbb{R}$ are expressed as a linear combination of $N$ basis filter responses. According to our opinion, steerability approaches may be classified in exact and approximate methods.

Although steerability was implicitly used by Danielsson and Knutsson [6], [10], Freeman and Adelson [9] were the first who coined the concept and introduced an exact steerability 
approach. Freeman's and Adelson's approach to orientation steerability of a Gaussian derivative is an interpolation using rotated copies of the original filter. The derived kernels do not possess sufficient orientational resolution due to their large spatial support in orientation. Moreover, they are either symmetric or antisymmetric [23]. This results in a period of $180^{\circ}$ in orientation and leads to an ambiguity in responses between terminating and nonterminating junctions.

Simoncelli et al. [24] extended this concept to include dilation and translation. They proposed some conditions upon which a filter is guaranteed to be exactly steerable, i.e., a filter can be synthesized with finite Fourier bases. Recently, Simoncelli and Farid [23] designed a steerable wedge filter in the inverse direction. Regarding the angular direction, they do not first choose one filter with expected shape and then project it onto the Fourier basis. Instead, they first choose finite components from the Fourier basis and then synthesize the filter using only these components. Therefore, their filter is guaranteed to be band limited and exactly steerable. Besides, the shape of the wedge filter can be adjusted to be arbitrarily narrow if we adopt adequate Fourier components. There is no more symmetric ambiguity applying a steerable wedge filter because the wedge kernel is asymmetric in the angular direction.

Michaelis and Sommer [13] and Teo and Hel Or [26] provided the formal justification of the exact steerability by applying Lie-group theory. The basic concept of exact steerability is the shiftability [24]: Every periodic band-limited function can be approximated at every position ("shift") with a finite linear combination of harmonic functions. Orientation and scaling become shifts if we apply a logarithmic-polar transformation to a function with two arguments. Lie group theory gives us the theoretical framework for this transformation and for the exact interpolation. The complex harmonics $e^{j \omega k}$ are the generating operators of translation. On the other hand, all one-parameter Lie groups are locally isomorphic to the translation group if we change the coordinates to so called canonical coordinates, for example, Cartesian to polar coordinates for rotations. Thus, for every deformation we achieve exact steerability if we transform the coordinates to canonical ones and then apply Fourier analysis. Teo and Hel Or list a complete classification of functions steerable with respect to any Abelian group.

Perona [17] introduced the concept of deformable kernels which is based on the minimization of the discrepancy between the left-hand side and the right-hand side of (1) with respect to the basis functions $A_{k}(\vec{x})$. He showed that the basis functions are the right singular functions of a continuous SVD. In case of rotations and periodic translations it can be proved [11] that the basis functions are the same in both the deformability and the exact Lie-group based steerability. However, for other deformations the functions must be sampled with respect to the deformation parameter and a numerical SVD is applied [25]. The advantage of the deformability approach is that it steers continuous and discrete filters and needs a minimal number of basis functions for a given error. However, orientational resolution is not addressed in this approach so that usually this approach suffers from the uncertainty constraint. Since we are interested only in orientation, both approaches yield the same set of basis functions.

The steerability problem may be also considered as a problem of signal reconstruction from samples $\gamma_{k}(k=1, \cdots, N)$ [see (2)]. This becomes evident if we consider the parameter $\alpha$ in (2) to be the same as the spatial domain variable. For clarity, we change the notation to $\theta$

$$
s(\theta)=\sum_{k=1}^{N} \gamma_{k} b_{k}(\theta) .
$$

Here, the interpolation functions $b_{k}(\theta)$ can be from many function classes, for example Laguerre functions or Legendre polynomials ([15, pp. 29-30]). In current orientation steerability approaches $b_{k}(\theta)$ are usually the complex harmonics $e^{j \omega_{k} \theta}$, yielding

$$
s(\theta)=\sum_{k=1}^{N} \gamma_{k} e^{j \omega_{k} \theta} .
$$

Note that $\omega_{k}$ are not necessarily the first $N$ frequencies as in the standard Fourier decompositions.

In this paper, we point out that the exact approach to orientation steerability has insufficient orientational resolution because it is based on the sampling of the angular frequency. To achieve a high orientational resolution a huge number of filters must be used. This computational burden is amplified by the large support of the basis functions. We will also introduce a new approximation approach based on Gaussian functions which might be nonoptimal with respect to the approximation error but substantially alleviates the above problems as shown in theory and experiments. We extend this approach to an efficient hierarchical scheme and provide a complete analysis of computational complexity. Thus, by considering only the problem of orientation steerability, we sacrifice a coherent algebraic theory (as in Lie group and SVD-based deformability approaches) to achieve high orientational resolution as well as a dramatical decreasing of the computational complexity.

This paper is organized as follows: In Section II we introduce the new approximate steerability approach based on angular Gaussians and point out the theoretical difference between our approach and current steerability approaches. Besides, we analyze their computational complexity in detail. In Section III we further introduce a polar pyramid scheme to treat orientation scale variations. Then we present experimental results on both synthetic and real data which vividly show a better performance of our approach. The paper is concluded with a short discussion.

\section{APPROXIMATE ORIENTATION STEERABILITY}

\section{A. Definition}

In the study of local orientation, we first conduct a local polar transformation of the image from Cartesian to the polar coordinate system and denote the new intensity function with $I(r, \theta)$, where $r$ and $\theta$ are the radius and angle, respectively. Since we are interested only in orientation, we eliminate the radial variable $r$ by applying averaging along the radial direction. In order to obtain high orientation resolution, we are interested in filters 

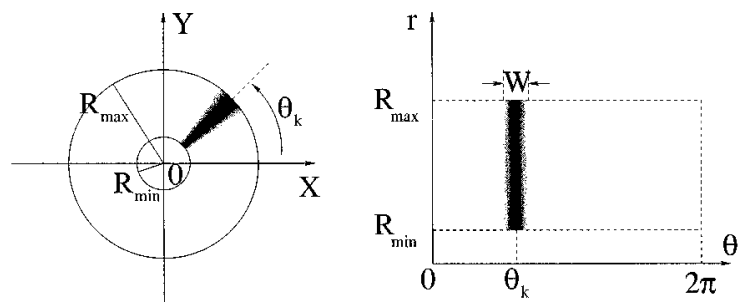

Fig. 1. Gaussian averaging mask centered at angle $\theta_{k}$. Darker pixels represent larger weights. Left: The mask in the Cartesian coordinate system. The keypoint is at the center of the circle. Right: The mask with $r$ and $\theta$ as coordinates, $R_{\max }$ and $R_{\min }$ are radial boundaries of the mask, $W$ is the angle width of the mask. We set $R_{\min }>0$ to avoid the confusion close to the keypoint.

with narrow angular support. We choose shifted Gaussian averaging functions as basis filters to sample the orientation space

$$
A_{k} \stackrel{\text { def }}{=} \frac{G_{0}\left(\mathcal{D}\left(\theta, \theta_{k}\right)\right)}{\mathcal{N}\left(R_{\min }, R_{\max }, \theta_{k}\right)}
$$

with $\theta_{k}(k=1, \cdots, M)$ distributed evenly along the axis of the orientation variable $\theta$. Here $\mathcal{N}\left(R_{\min }, R_{\max }, \theta_{k}\right)$ is an averaging factor along the radial direction which is the sum of discrete weights inside the basis filter mask centered at $\theta_{k}$ (see Fig. 1). We denote with $G_{0}\left(\mathcal{D}\left(\theta, \theta_{k}\right)\right)$ the Gaussian function centered at $\theta_{k}$

$$
G_{0}\left(\mathcal{D}\left(\theta, \theta_{k}\right)\right) \stackrel{\text { def }}{=} \frac{1}{\sqrt{2 \pi} \sigma} e^{-\left(\mathcal{D}\left(\theta, \theta_{k}\right)\right)^{2} / 2 \sigma^{2}}
$$

where $\sigma$ denotes the scale of the Gaussian function. Since $\theta$ and $\theta_{k}$ are circular angles $\left(\theta, \theta_{k} \in[0,2 \pi]\right)$, we define $\mathcal{D}(\cdot)$ to represent the minimal circular difference between $\theta$ and $\theta_{k}$

$$
\mathcal{D}\left(\theta, \theta_{k}\right) \stackrel{\text { def }}{=} \min \left(\left|\theta-\theta_{k}\right|,\left|\theta-\theta_{k}-2 \pi\right|,\left|\theta-\theta_{k}+2 \pi\right|\right) \text {. }
$$

For example, $\mathcal{D}(2 \pi, 0)=0 ; \mathcal{D}(359 \pi / 180, \pi / 180)=\pi / 90$. Theoretically, a Gaussian function is not compactly supported. In practice, we only consider the part of $G_{0}\left(\mathcal{D}\left(\theta, \theta_{k}\right)\right)$ whose variable varies from $\theta_{k}-(W / 2)$ to $\theta_{k}+(W / 2)$ [see (11)]. Here, $W$ denotes the angular width of the basis filter mask. We will explain the choice of parameter $W$ in Section II-C. In Fig. 1, we show a basis filter centered at $\theta_{k}$, where $R_{\min }$ and $R_{\max }$ denote inner and outer boundaries of the mask, respectively. We set $R_{\min }>0$ to avoid the singularity close to the keypoint [12]. In order to choose $R_{\max }$ we must know the size of the significant neighborhood around the keypoint, which can be provided by the preceding keypoint detection step [8], [20]. In this paper we set $R_{\min }=3$ pixels and $R_{\max }$ varies from nine to 15 pixels.

After defining basis filters we must interpolate them to build the whole set of steerable filter $g(\theta)$. Taking the local property of basis filters into account we choose angular Gaussian function with narrow support instead of complex harmonics as the interpolation function

$$
b_{k}(\theta) \stackrel{\text { def }}{=} G_{0}\left(\mathcal{D}\left(\theta, \theta_{k}\right)\right) .
$$

Thus, we construct a continuous steerable filter using Gaussian interpolation functions

$$
g(\theta) \stackrel{\text { def }}{=} \sum_{k=1}^{M} A_{k} G_{0}\left(\mathcal{D}\left(\theta, \theta_{k}\right)\right) .
$$
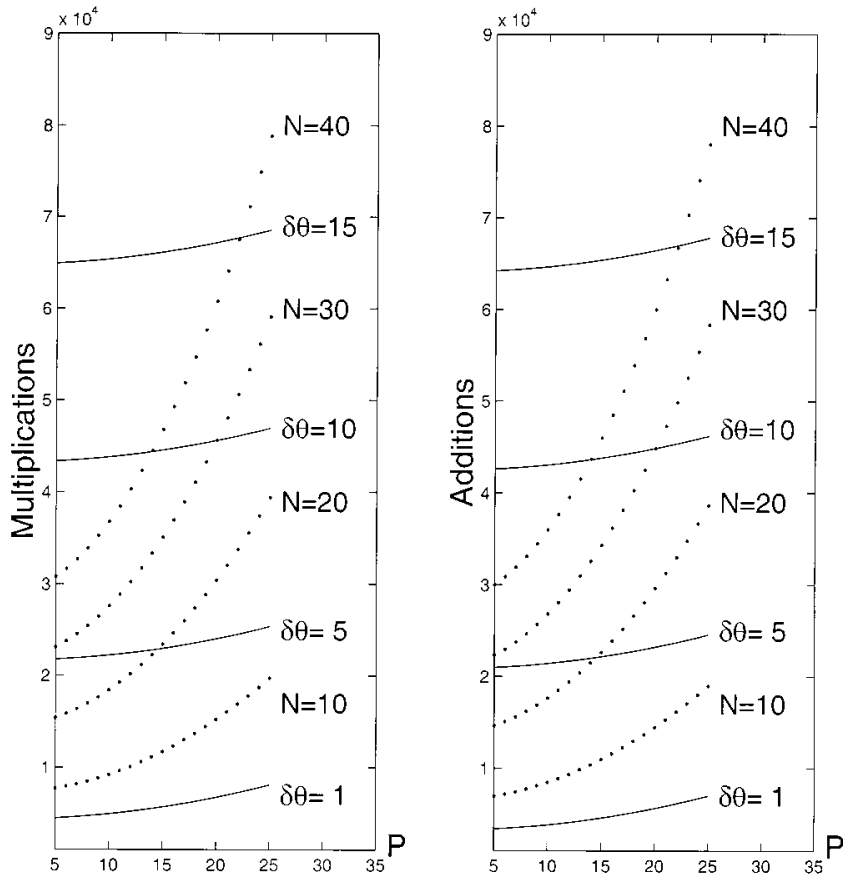

Fig. 2. Complexity comparison between the exact steerability and the approximate steerability. The dotted curves represent the complexity of exactly steerable filters composed of $N$ odd basis filters and $N$ even basis filters with $P$ as 1-D tap size. The solid lines show the relation between complexity and the sampling interval $\delta \theta$ and $P$ in computation of $S$ and $D S$ in our approach. We see that the approximate steerability is more efficient than the exact steerability, especially in the case of high orientation resolution and large filter size.

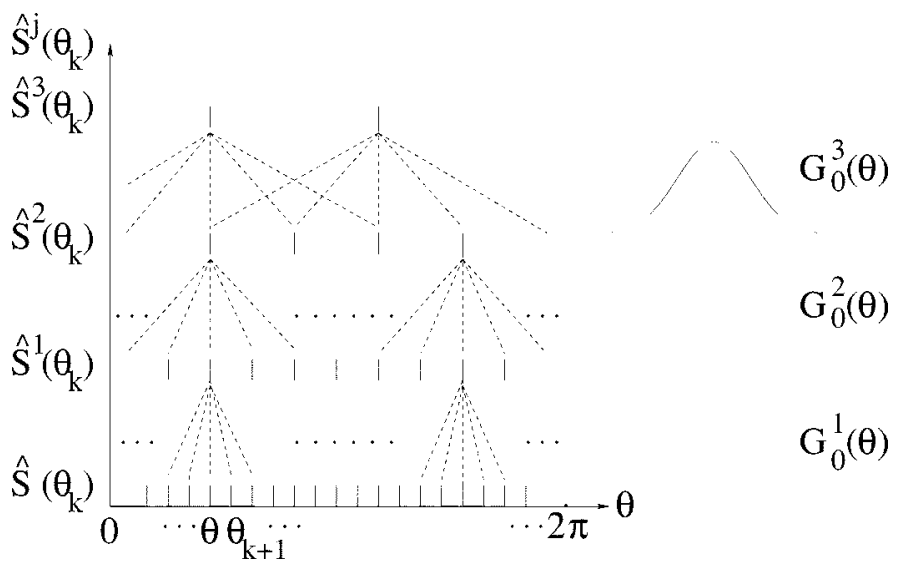

Fig. 3. Left: Polar pyramid structure, $S(\theta)$ is the lowest level of the polar pyramid, namely the averaging outputs of sampling masks. $\hat{S}^{j}\left(\theta_{k}\right)(j=1,2,3)$ are higher levels after subsampling with the generating kernel $K_{2}$ of (22). Right: Corresponding interpolation functions at different levels. They are Gaussian functions with different scales.

In this new steerability approach, the neighboring basis filters as well as the interpolation functions are locally correlated and therefore nonorthogonal. To achieve optimal steerability we would have to apply a nonlinear operator to estimate the coefficients of the interpolation function. Instead we use a linear operator and achieve an approximate steerability which approaches optimality with decreasing support and thus increasing orthogonality of the Gaussians. Approximating a function with a sum of Gaussians is a well-known method with properties extensively described in the radial basis function approximation proposed by Poggio and Girosi [19]. If we ignore the penalty term in 

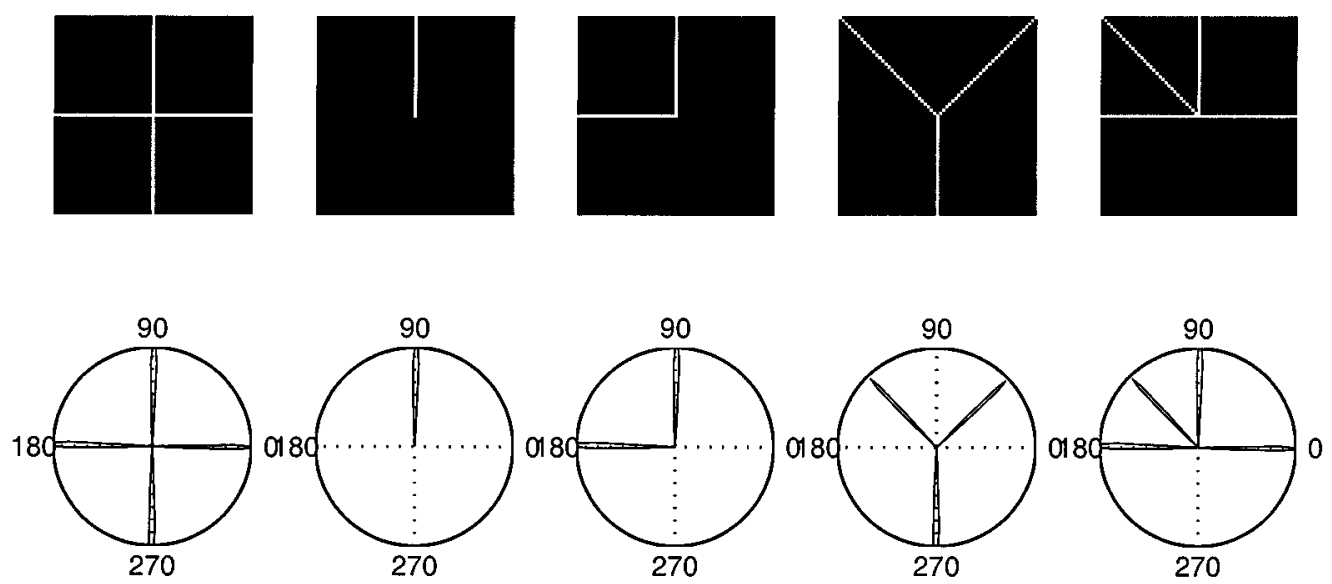

Fig. 4. Top: Synthetic line junctions. Bottom: $S(\theta) . R_{\min }=3, R_{\max }=15$.

[19] enforcing smoothness our approach becomes the closer to the RBF approach when our Gaussians overlap minimally each other. To increase approximation optimality the centers of the Gaussians can be estimated in [19] whereas in our approach these centers are prechosen.

Applying our filter on the intensity function $I(r, \theta)$ we obtain the orientation signature $S(\theta)$

$$
S(\theta) \stackrel{\text { def }}{=} \sum_{k=1}^{M} \gamma_{k} G_{0}\left(\mathcal{D}\left(\theta, \theta_{k}\right)\right)
$$

where

$$
\begin{aligned}
\gamma_{k} \stackrel{\text { def }}{=} & \sum_{\theta=\theta_{k}-(W / 2)}^{\theta_{k}+(W / 2)} G_{0}\left(\mathcal{D}\left(\theta, \theta_{k}\right)\right) \\
& \cdot \sum_{r=R_{\min }}^{R_{\max }} \frac{I(r, \theta)}{\mathcal{N}\left(R_{\min }, R_{\max }, \theta_{k}\right)} .
\end{aligned}
$$

In the orientation signature $S(\theta)$ local extremes represent orientations of lines and the positions of steepest descent or ascent indicate orientations of edges. Correspondingly, the two-dimensional (2-D) orientation analysis reduces into 1-D line/edge detection. In order to extract edge information we estimate the derivative of $S(\theta)$ and take the amplitude of the derivative as another signature

$$
\begin{aligned}
& D S(\theta) \stackrel{\text { def }}{=}\left|\frac{d}{d \theta} S(\theta)\right| \\
&=\left|\sum_{k=1}^{M} \gamma_{k} \frac{d}{d \theta} G_{0}\left(\mathcal{D}\left(\theta, \theta_{k}\right)\right)\right| \\
&=\left|\sum_{k=1}^{M} \gamma_{k} G_{1}\left(\mathcal{D}\left(\theta, \theta_{k}\right)\right)\right|
\end{aligned}
$$

where $G_{1}\left(\mathcal{D}\left(\theta, \theta_{k}\right)\right)$ denotes the first derivative of Gaussian filter $G_{0}$ and $|\cdot|$ denotes the absolute value. We point out here that $S(\theta)$ and $D S(\theta)$ cannot constitute a quadrature pair because $D S(\theta)$ is not the Hilbert transform of $S(\theta)$. Such a pair would have significant energy on the negative frequencies and the estimated magnitude would be phase-dependent. To characterize junctions we are not interested in a phase-independent magnitude response because we want to know which orientation responses are closer to an edge and which are closer to line. Therefore, we obtain two separate signatures from $S(\theta)$ and $D S(\theta)$, for lines and edges, respectively.

So far, we have defined the approximate steerability. In the following we will compare this approach with current steerability approaches regarding the mathematical background and the implementation performance.

\section{B. Difference to Exact Steerability}

The main difference between our approach and the exact steerability is that we decompose one signal locally in the spatial domain, whereas exact approaches decompose the signal globally: In the approximate steerability we use a Gaussian mask with local spatial support (as shown in Fig. 1) as basis filter [i.e., $A_{k}$ in (5)], while in the exact steerability every basis filter has the same wide spatial support as the steered filter. This difference can be described in the spectral domain as well. In the approximate steerability the spectrum of a filter response [see (10)] is decomposed into a set of spectral Gabor functions (shifted Gaussians in the spatial domain) weighted by the basis filter responses $\gamma_{k}$

$$
\begin{aligned}
\mathcal{F}[S(\theta)] & =\sum_{k=1}^{M} \gamma_{k} \mathcal{F}\left[G_{0}\left(\theta-\theta_{k}\right)\right] \\
& =\sum_{k=1}^{M} \gamma_{k} \mathcal{F}\left[G_{0}(\theta)\right] e^{-j \omega \theta_{k}}
\end{aligned}
$$

where $\mathcal{F}$ denotes the Fourier transform. In contrast, in the exact steerability the spectrum of a filter response [see (4)] is decomposed into a series of Dirac sampling functions weighted by the corresponding basis filter responses

$$
\mathcal{F}[s(\theta)]=\sum_{k=1}^{N} \gamma_{k} \delta\left(\omega-\omega_{k}\right) .
$$

This formula describes also the behavior of the exact approaches using rotated filter copies as basis functions. The proof of the steerability using rotated copies is based on the fact that 

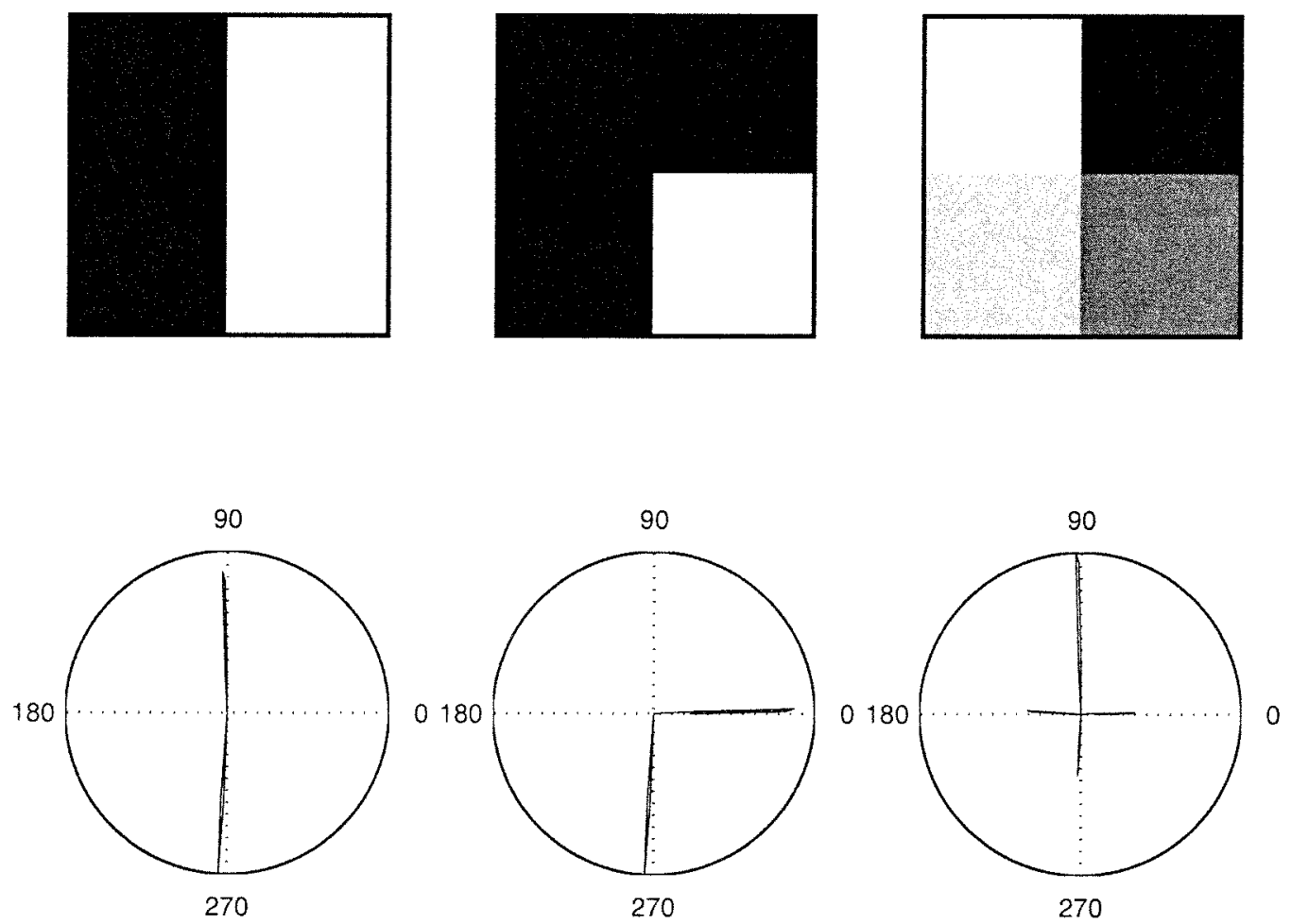

Fig. 5. Top: Synthetic edge junctions. Bottom: $D S(\theta)$. The local maxima show the orientation of edges. $R_{\min }=3, R_{\max }=15$.
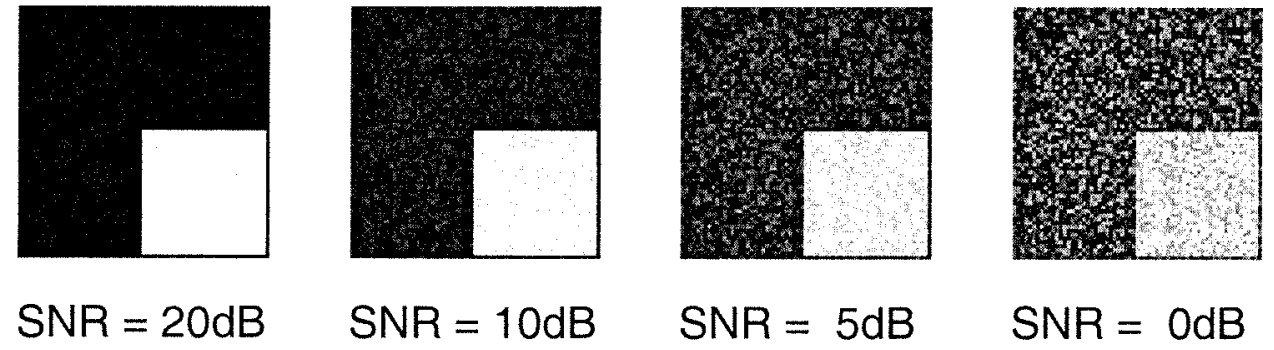

$\mathrm{SNR}=\mathrm{OdB}$
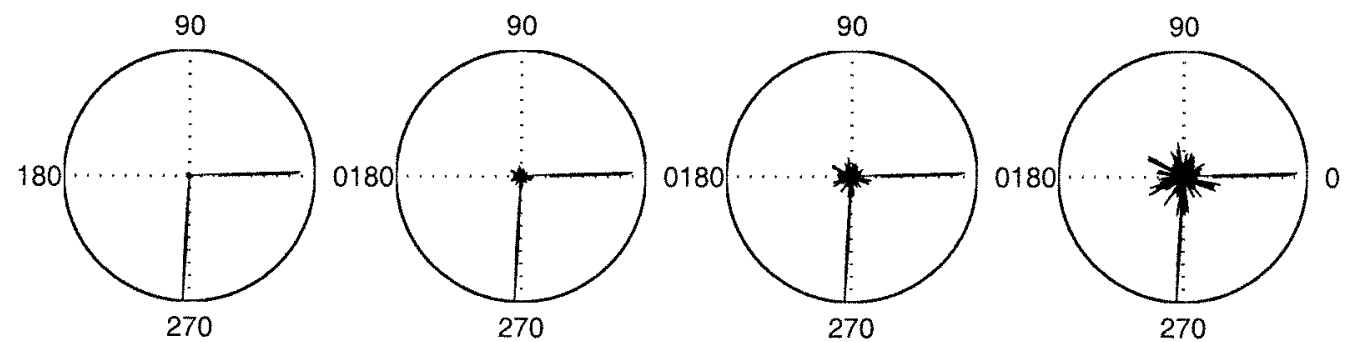

Fig. 6. Top: A synthetic edge junction disturbed by four increasing levels of random noise. Bottom: Corresponding $D S(\theta)$. Even in the very noisy case (SNR $=0$ $\mathrm{dB})$ the signature can characterize the junction. $R_{\min }=3, R_{\max }=15$.

a function is written as a Fourier series with respect to the angle (see [9, eq. (9)]). Equation (14) is the expression of this fact in the frequency domain. Note that in (14) the term $a_{n}(r)$ in [9] is replaced by $\gamma_{k}$ since we consider the filter response here.

Thus, in the case of approximate steerability we sample the spatial orientation space with Gaussian masks whereas in the exact steerability we sample the spectral domain of the orientation space with Dirac sampling functions. This difference makes our approach perform better with respect to orientational resolution. According to the well known uncertainty principle we cannot localize one signal both in the spatial domain and in the spectral domain exactly. If we use one Dirac sampling function to localize one spectral component of the signal exactly, as in the case of the exact orientation steerability, we will no more be able to localize this component in the spatial domain. Therefore, we need many Dirac impulses to increase the localization capability in space. This tradeoff can be optimized by applying functions with Gaussian shape [7]. Therefore, the approximate steerability has better properties with respect to the uncertainty principle.

To summarize, our main concern is orientational resolution with low complexity. To achieve this goal, we directly built our 

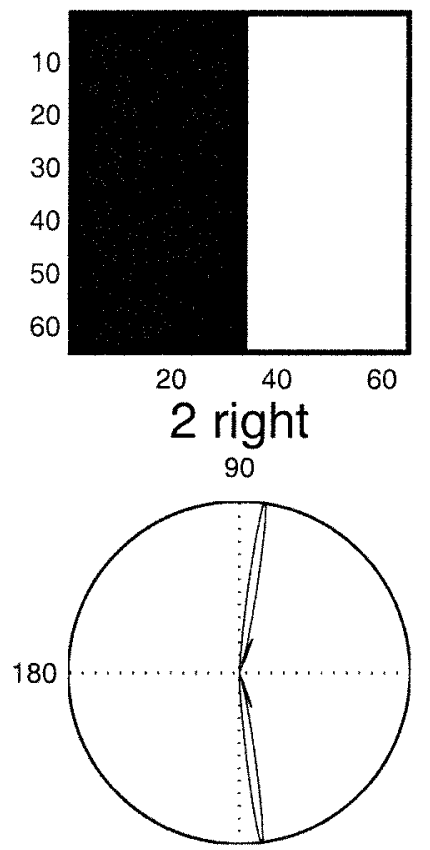

270
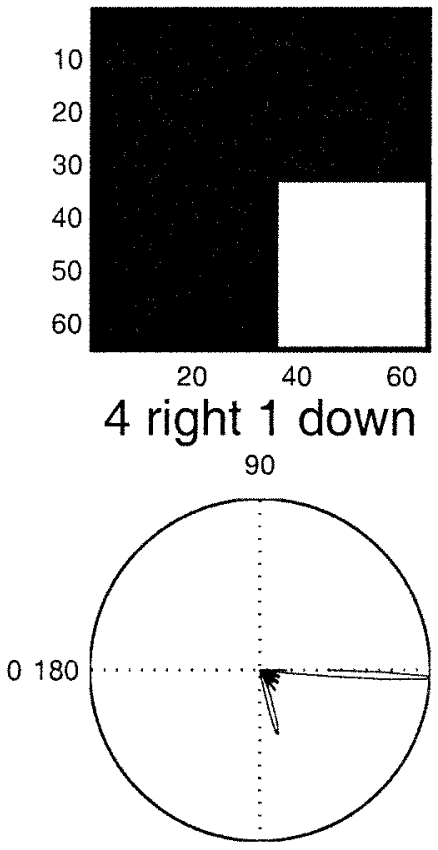

270

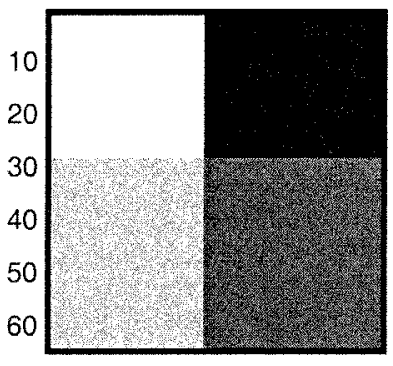

$20 \quad 40 \quad 60$

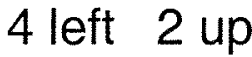

90

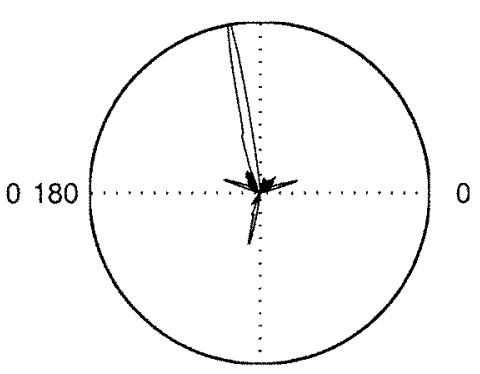

270

Fig. 7. Top: Deviation of the keypoint from the central position of the mask. Bottom: $D S(\theta) . R_{\min }=3, R_{\max }=15$.

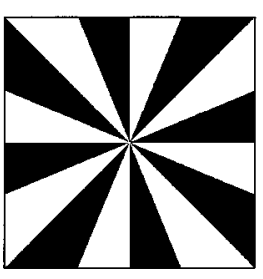

A: Star

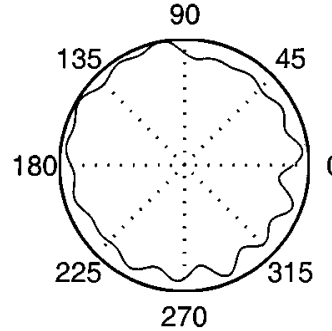

B: $2 \mathrm{~N}=46$

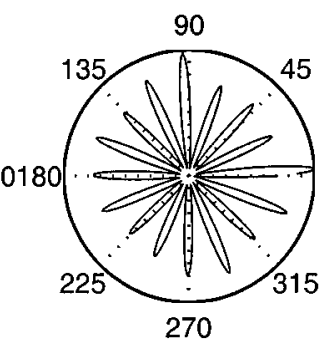

C: $2 \mathrm{~N}=90$

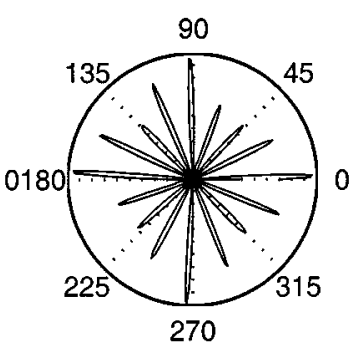

D: $\mathrm{DS}(\theta)$

Fig. 8. (a) So-called "Siemens star" with 16 edges spanning uniformly the orientation space. (b) Polar plot of the result using the steerable wedge filter [23] composed of 46 basis filters with 31-tap size. In total, 61486 multiplications and 60360 additions are needed. The edges are hardly discernible. (c) The same as (b) but using 90 basis filters with 119610 multiplications and 118440 additions. The orientations of the edges are clearly presented. (d) $D S(\theta)$ using the Gaussian averaging steerable filter. We compute only 10086 multiplications and 9006 additions to achieve the same resolution.

filter in the spatial domain. The price we pay is that we do not achieve exact steerability but an approximation of the orientation response.

\section{Complexity Analysis}

Approximate steerability achieves a higher orientation resolution with a lower complexity due to the narrow support of the basis filters. Our approach starts with a local polar mapping which can be done "off-line" since it is a transform between coordinates and is therefore valid for all different images. Online applying the resulting look-up-table (LUT) is of negligible complexity compared with calculating the filter responses.

In order to compare the implementation complexity of the approximate steerability and that of the exact approach we set the radial extensions of masks in both schemes to be the same.
If we denote the 1-D tap size of an exactly steerable filter with $P$, the following relation is satisfied:

$$
P=2 R_{\max }+1 .
$$

Here, we choose the steerable wedge filter [23] for comparison since it has a similar shape to our filter mask. The steerable wedge filter is a separable polar filter. Its radial component looks like a wedge and its angular component is synthesized by the Fourier series. Its computational complexity is proportional to the number and the spatial support of basis filters. In order to make a fair comparison we apply it after the local polar mapping, too.

Both the approximately steerable filter and the wedge filter are polar separable filters. If we carry out a polar mapping on the images then filter responses can be computed with two 1-D 


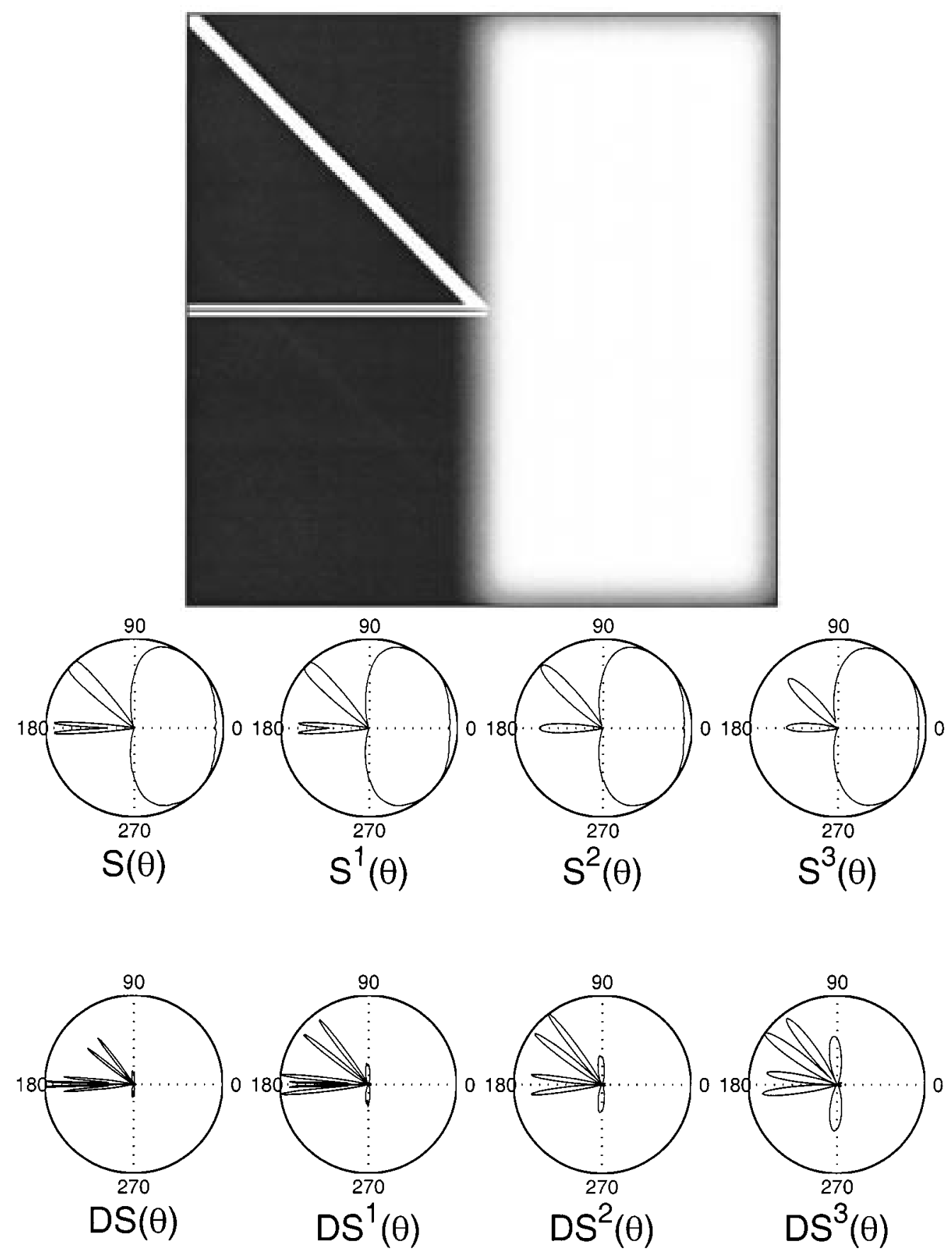

Fig. 9. Characterizing a junction using the polar pyramid. Top: A junction composed of two close lines with distance of 1 pixel, two edges blurred by a Gaussian function with $\sigma=4$ and a wide line with a width of 6 pixels. Middle: The characterizing signatures reconstructed from the first four levels of the polar pyramid: $S(\theta), S^{1}(\theta), S^{2}(\theta)$, and $S^{3}(\theta)$. The wide line is distinctly characterized in $S^{3}(\theta)$. But two close lines are also recognized as one line. Bottom: Corresponding $D S(\theta), D S^{1}(\theta), D S^{2}(\theta)$, and $D S^{3}(\theta)$. The blurred edges are presented more and more clearly with the increase of pyramid level. The neighboring boundaries of two close lines near $180^{\circ}$ can be seen only at the first two levels. After increasing the scale only outer boundaries of these two lines are recognized.

convolutions in radius and angle, respectively. Such a pre-mapping would have the effect that the complexity of both filters would depend linearly and not quadratically on the tap size $P$. Because we deal in this paper with characterization and not with detection we assume that several candidate points have been detected and that we do not need a signature at every image position. Therefore, it is more economical to compute the filter response at specified positions using a scalar product though its complexity is quadratic on the tap size $P$. When comparing with the wedge filter the reader should have in mind that, if convolution were applied, approximate filter would still perform better but only linearly and not quadratically.

Suppose we apply a steerable wedge filter composed of $2 N$ basis filters ( $N$ odd basis filters and $N$ even basis filters). As mentioned before, all basis filters have the same spatial support as the steered filter, the 1-D size of all basis filters is therefore $P$, too. In order to apply one basis filter we need $P^{2}$ multiplications and $P^{2}-1$ additions to calculate the corresponding 

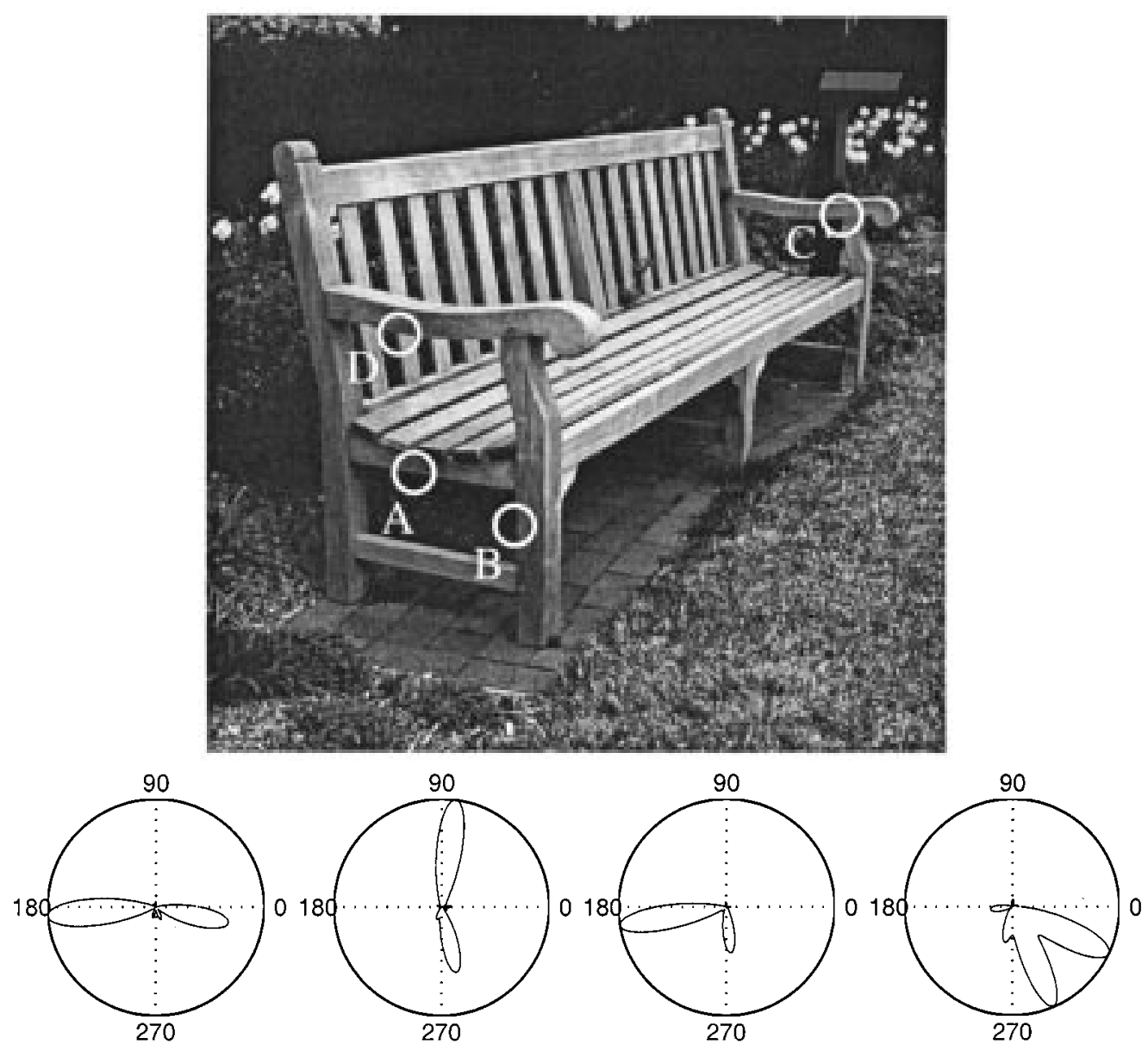

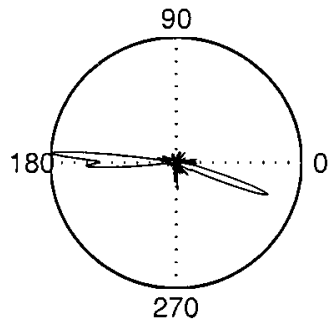

A

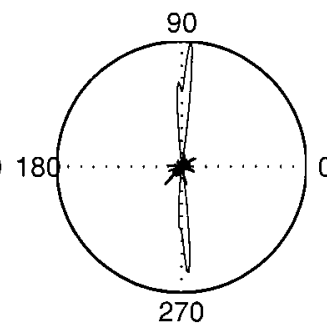

B

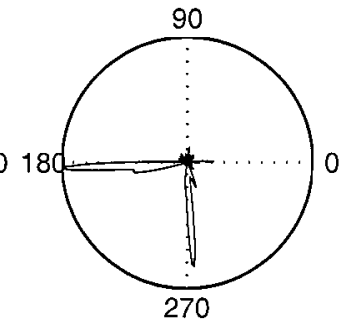

C

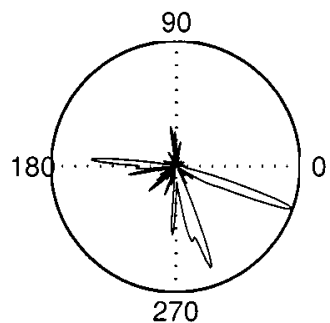

D

Fig. 10. Top: The parkbench with marked edge junctions. A: horizontal edge; B: vertical edge; C: corner; D: “ $T$ "'-junction. Middle: Steerable wedge filter results using 30 basis filters. $P=19$. Bottom: $D S(\theta)$ of the Gaussian averaging steerable filter. The edge near $180^{\circ}$ in $\mathrm{D}$ is very blurred. But $D S(\theta)$ still can characterize it. $R_{\min }=3, R_{\max }=9$.

inner product. Straightforwardly, $2 N P^{2}$ multiplications and $2 N\left(P^{2}-1\right)$ additions are required to obtain $2 N$ coefficients [see (2)]. Assuming that we use signatures of length 360 we need $720 N$ multiplications and $720(N-1)$ additions to obtain outputs of odd and even filters. Thus, totally $2 N\left(P^{2}+360\right)$ multiplications and $2 N\left(P^{2}+359\right)$ additions are computed to implement a steerable wedge filter composed of $N$ odd basis filters and $N$ even basis filters.

In our approach the computational load is determined by the number and the angular width of Gaussian functions. In order to sample the whole orientation space with a sampling interval $\delta \theta$, we need totally $M=360 / \delta \theta$ basis filters. According to the well known Shannon's Sampling Theorem, we determine the corresponding Nyquist frequency $f_{m}$ with

$$
f_{m}=\frac{1}{2 \delta \theta} .
$$

In our approach, we choose Gaussian masks instead of Dirac functions as sampling masks along the angular direction. This is equivalent to Dirac series [the shah function $\operatorname{III}(\theta)$ [4]] convolved with a Gaussian function. Correspondingly, the spectrum of the ideal sampling will be further multiplied by a low pass filter with Gaussian shape. The stop frequency of this low pass filter is determined by the term $1 / 2 \sigma$ (here we define the turning 

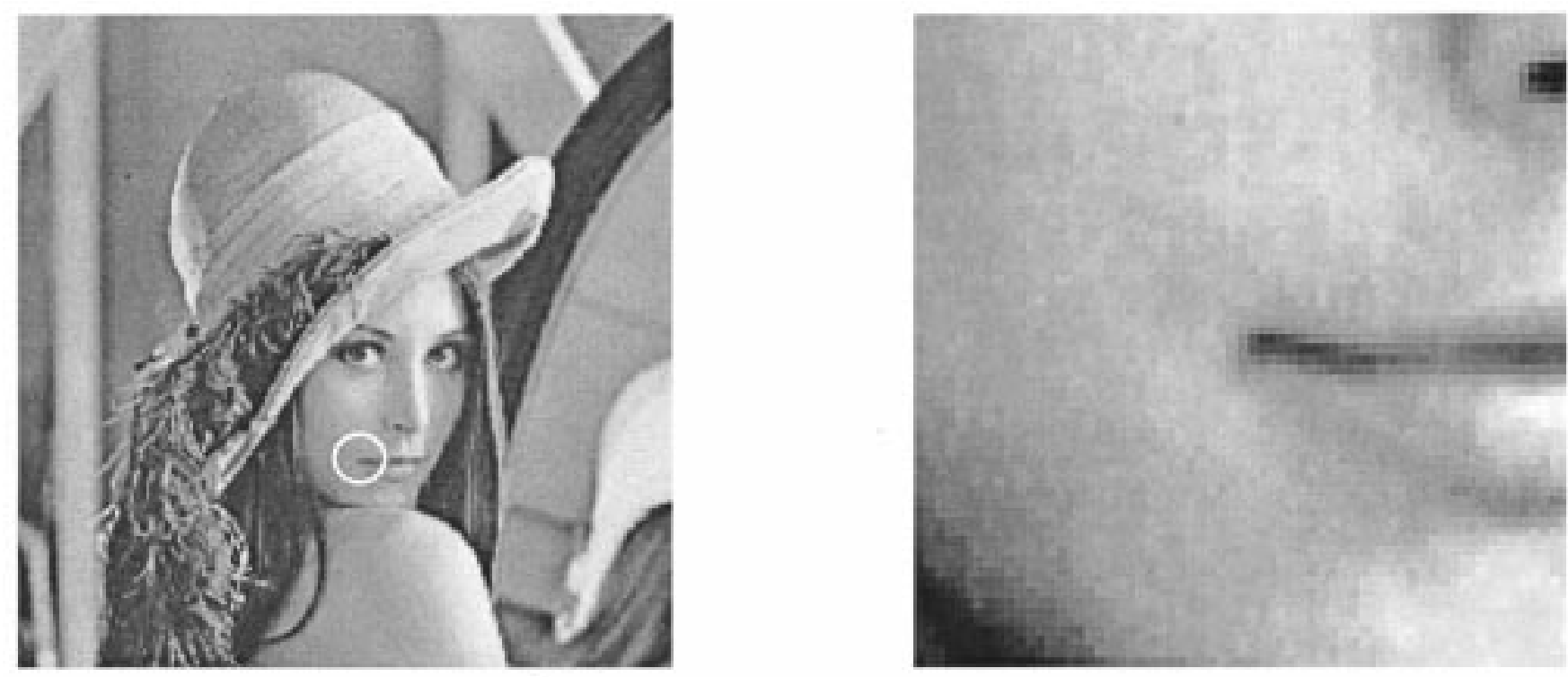

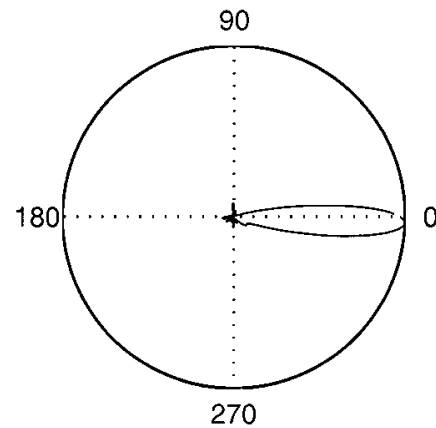

$2 \mathrm{~N}=46$

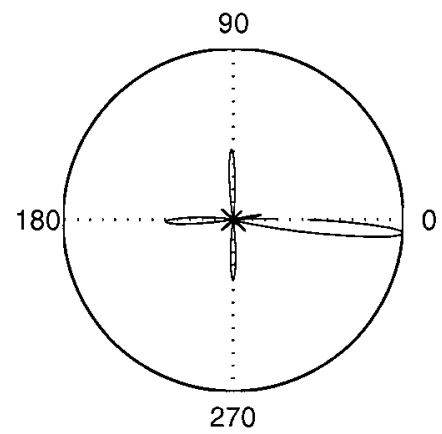

$2 \mathrm{~N}=90$

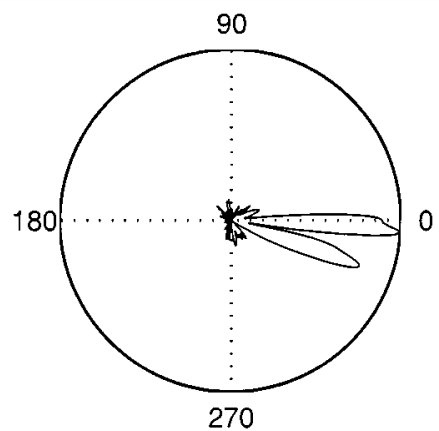

$\mathrm{DS}(\theta)$

Fig. 11. Resolution comparison between the steerable wedge filter [23] and the Gaussian averaging steerable filter. Top Left: The image "Lena" with her lips corner as a keypoint. Top Right: Lips corner in detail. Bottom Left: Polar plots using the steerable wedge filter [23] with 46 basis filters. Only the dominant dark line between the lips can be recognized. Bottom Middle: Even with 90 basis filters we cannot recognize two lips. Bottom Right: $D S(\theta)$ of the Gaussian averaging steerable filter. The edges of two lips are characterized distinctly.

frequency of the Gaussian function $1 / 2 \sigma$ as the stop frequency). This stop frequency is preferred to be not below the Nyquist frequency $f_{m}$

$$
\frac{1}{2 \sigma} \geq f_{m}=\frac{1}{2 \delta \theta}, \quad \text { hence } \quad \sigma \leq \delta \theta .
$$

On one hand, we should set the scale parameter $\sigma$ small in order to have fine orientation resolution. On the other hand, the filter mask should contain adequate pixels to calculate averaging values robustly. Therefore, we set $\sigma=\delta \theta=1^{\circ}$ to achieve the compromise between fine resolution and robustness. Theoretically, a Gaussian function is not compactly supported. Thus, we must cut off its support to build an FIR-filter. It is easy to show that in order to keep the energy of the cut-off area below $1 \%$ of the total energy, the angular width of the sampling mask $W$ must be at least $5 \sigma$. In this paper, we set $W=6 \sigma$.

We use $M=360 / \delta \theta$ sampling masks to obtain angular samples $\gamma_{k}(k=1, \cdots, M)$. On an average we have at most $(W / 360) \pi\left(R_{\max }^{2}-R_{\min }^{2}\right)<(W / 360) P^{2}$ pixels in one sampling mask. In order to analyze the upper limit of the complexity, we simply assume that in one sampling mask there are $(W / 360) P^{2}$ pixels. Correspondingly, we need $(W / 360) P^{2}$ multiplications and $(W / 360) P^{2}-1$ additions to calculate one angular sampling. Taking into account that $W=6 \delta \theta$ we need $6 P^{2}$ multiplications and $6 P^{2}-(360 / \delta \theta)$ additions to obtain $M$ samples. In constructing $S(\theta)$ and $D S(\theta)$ we apply a Gaussian function and its first derivative, respectively, with the angular width $W$ as interpolation functions. In order to produce the signatures $S(\theta)$ and $D S(\theta)$ with the same length of 360 as in the wedge filter approach above, we require $360 \mathrm{~W}$ multiplications and $360(W-1)$ additions for interpolation. Thus, totally we need $6 P^{2}+4320 \delta \theta$ multiplications and $6 P^{2}+4320 \delta \theta-(360 / \delta \theta)-720$ additions to obtain $S(\theta)$ and $D S(\theta)$ in case of approximate steerability.

The complexity of both approaches is plotted in Fig. 2. In the approximate steerability the sampling interval $\delta \theta$ plays a very interesting role. We observe that by setting $\delta \theta$ smaller, which means we have higher orientation resolution, computation load decreases. We can draw the conclusion that the approximate steerability is more efficient than the exact steerability, especially in the case of high orientation resolution and large filter size.

\section{JUNCTION CHARACTERIZATION USING A POLAR PYRAMID}

The orientation scale problem is like every scale problem a tradeoff between the intrinsic structure of a junction and the orientation scale of a filter. As shown in Fig. 9, if the orientation 

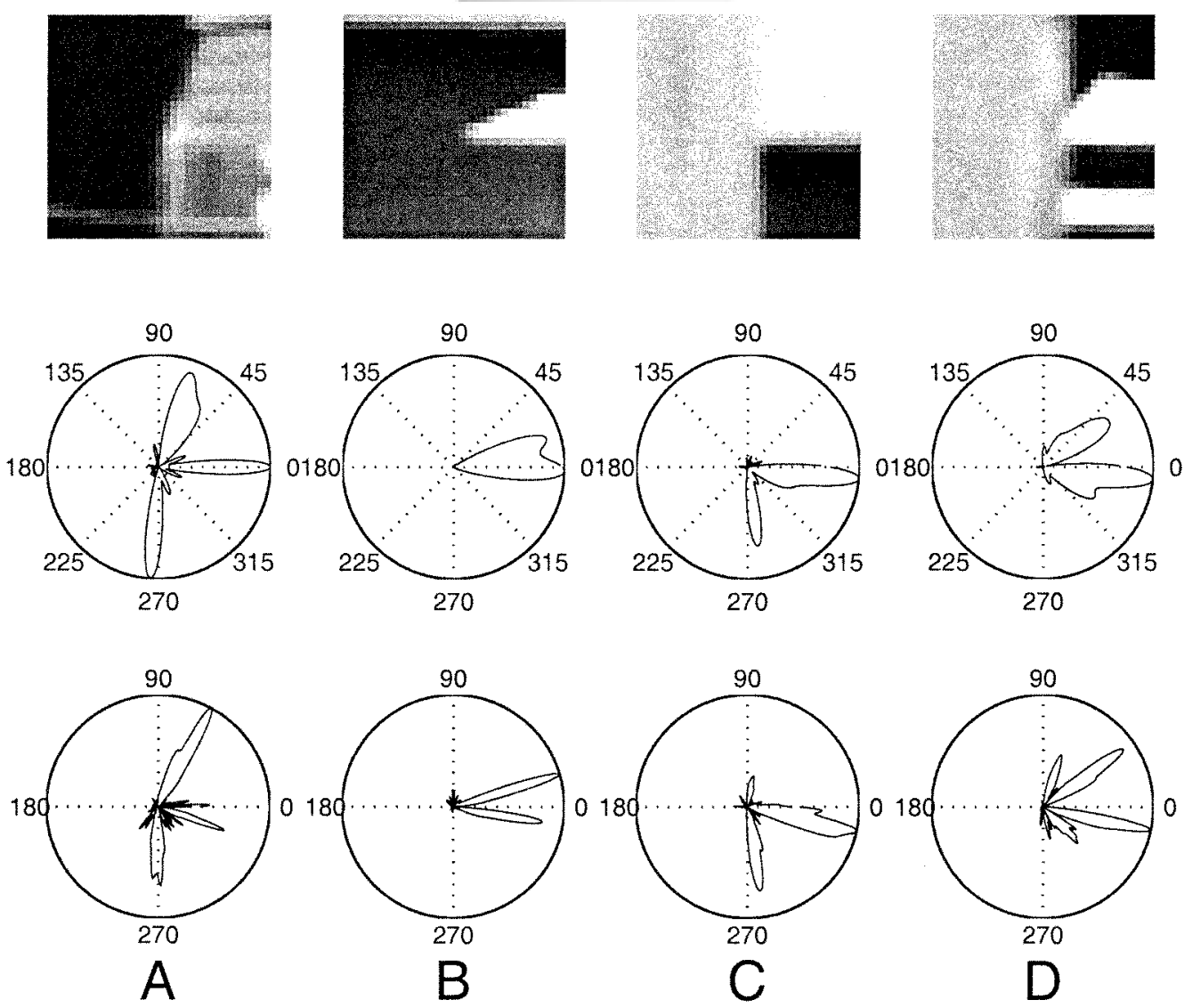

Fig. 12. Comparison between the steerable wedge filter [23] and Gaussian averaging steerable filter. Row 1: An image of the NASA sequence with four kinds of marked junctions. A: " $Y$ " junction; B: " $V$ " junction; C: " $T$ " junction; D: " $K$ " junction. Row 2: Junctions in detail. The keypoints are not always at centers of the masks. Row 3: Polar plots using the steerable wedge filter [23] using 46 basis filters with 31 tap size. Row 4: $D S(\theta)$ using the Gaussian averaging steerable filter $\left(R_{\min }=3, R_{\max }=15\right)$. Both methods are stable with respect to the offsets of keypoints. Our method presents higher orientation resolution with lower cost.

scale of a filter is too small, a blurred edge is not visible and a wide line will be recognized as two edges [12]. If the orientation scale of a filter is too large, two very close lines will be characterized as one line. In this paper we introduce a polar pyramid to obtain signatures of different scales efficiently. Here we do not treat the problem of steering spatial scale. Regarding the steerability of spatial scale the reader is referred to [11], [18].

It is known that one of the most appealing kernels for hierarchical approaches is the Gaussian function [1]. We know from spatial scale theory that the choice of scale necessitates a hierarchical treatment. Burt and Adelson [5] prove that a generating kernel of subsampling can be used as the interpolation function for reconstruction from coarser scales. Moreover, they argue that the interpolation functions can be (discrete approximations of) Gaussian functions with different scales (Fig. 3). Thus, the continuous orientation information can be reconstructed from all levels of the polar pyramid by interpolating the pyramid el- ements $\hat{S}^{j}\left(\theta_{k}\right)$ with Gaussian functions $G_{0}^{j}\left(D\left(\theta, \theta_{k}\right)\right)$ of different scales $\sigma_{j}$

$$
S^{j}(\theta)=\sum_{k} \hat{S}^{j}\left(\theta_{k}\right) G_{0}^{j}\left(D\left(\theta, \theta_{k}\right)\right) \quad j \in[1,2, \cdots]
$$

with

$G_{0}^{j}\left(D\left(\theta, \theta_{k}\right)\right)=\frac{1}{\sqrt{2 \pi} \sigma_{j}} e^{-\left(\left(D\left(\theta, \theta_{k}\right)\right)^{2} / 2 \sigma_{j}^{2}\right)} \quad j \in[1,2, \cdots]$

where $\hat{S}^{j}(\theta)$ denote the elements on the $j$ th level of the polar pyramid and $S^{j}(\theta)$ represents the signature reconstructed from $\hat{S}^{j}(\theta)$. We can obtain edge signatures similarly

$$
D S^{j}(\theta)=\left|\sum_{n} \delta(\theta-n \triangle \theta) \hat{S}^{j}(\theta) * G_{1}^{j}(\theta)\right|
$$



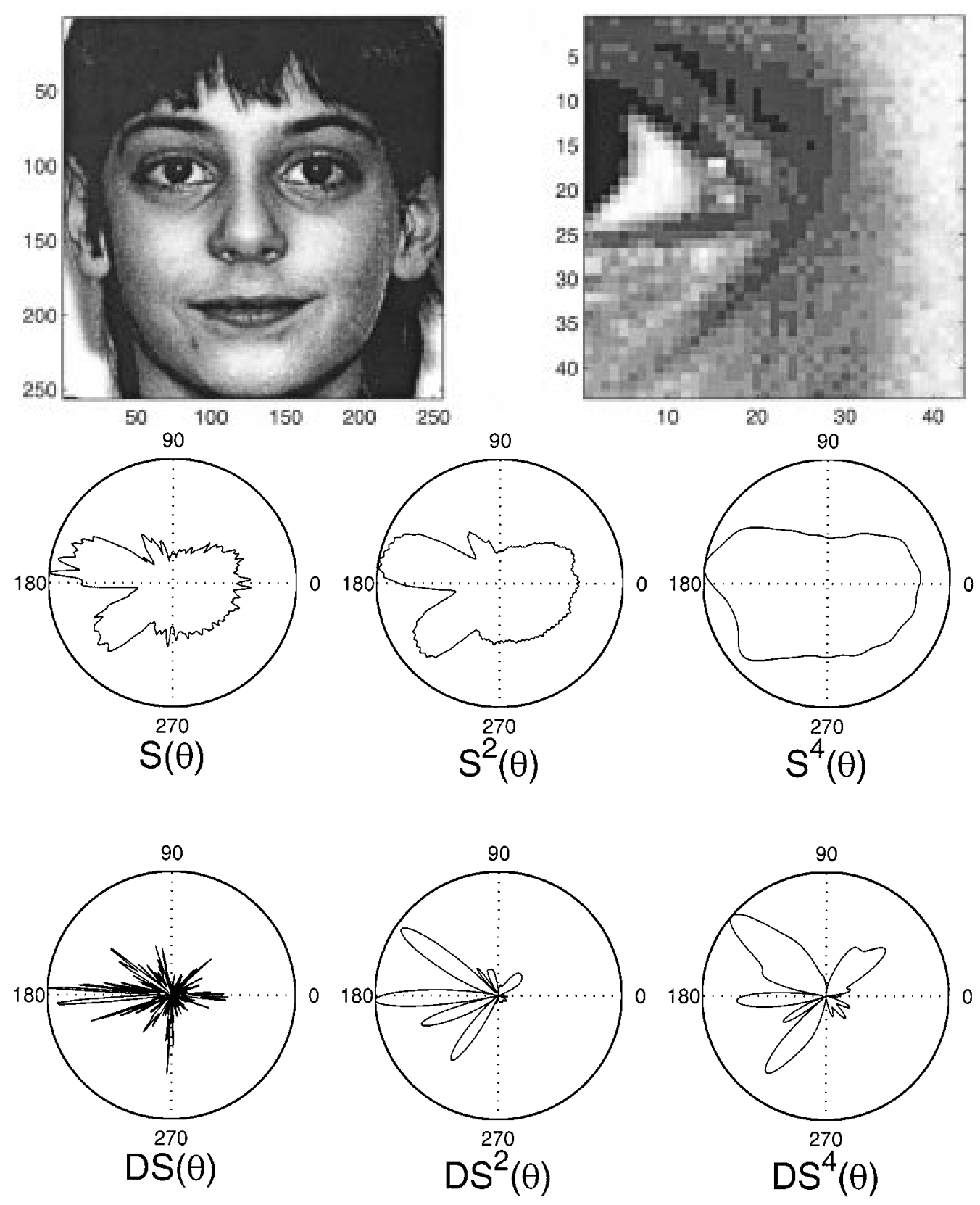

Fig. 13. Top Left: Face of a child. Top Right: Its left eye corner in detail. It can be regarded as a combination of irregular wide lines and blurred edges disturbed by noise. Middle: Orientation signatures reconstructed from the first, third and fifth level of the polar pyramid. The eyelids are clear to see in $S^{2}(\theta)$ as two maxima at $135^{\circ}$ and $225^{\circ}$. The local maximum near $180^{\circ}$ is due the white of the eye. Bottom: Corresponding $D S(\theta), D S^{2}(\theta)$ and $D S^{4}(\theta)$. At small scales the signatures present more detail, but are also sensitive to noise. At large scales we obtain dominant structures but lose details. A valid characterization should combine all these signatures.

where $G_{1}^{j}(\theta)$ is the first derivative of $G_{0}^{j}(\theta)$. The local maxima in $S^{j}(\theta)$ and $D S^{j}(\theta)$ denote orientations of lines and edges at different scales, respectively.

If we want to build a pure 1-D one-octave Gaussian pyramid of angles, according to [5] we should have $Q 2^{L}+1$ samples where $L$ is the number of levels and $Q+1$ is the number of samples at the highest level. Taking the periodicity into account we should have $Q 2^{L}$ samples as the sampling outputs. However, since initially the orientation signal is defined as 360 discrete values we cannot build a pure octave Gaussian pyramid. Alternatively, we apply a factor two subsampling of the first three levels, a factor three at the next two, and have five samples at the coarsest level $\left(360=2^{3} \times 3^{2} \times 5\right)$. Let us denote with $K_{i}(j)$ the $j$-th coefficient of the FIR filter in the $i$ th pyramid layer. According to [5] the generating kernels with subsampling factor $i$ should be normalized

$$
\sum_{j=1}^{2 i+1} K_{i}(j)=1 \quad i \in[2, \cdots]
$$

and symmetric

$$
K_{i}(j)=K_{i}(2 i+1-(j-1)) \quad j \in[1, \cdots, i+1] .
$$


Moreover, Every sample at a given level should make equal contributions to construct the next higher level. The contributions of one sample are weighted by the corresponding coefficients of the generating kernels. Equal contributions imply that the sum of all its connected elements in the generating kernels should be a constant.

As mentioned in [5], the generating kernels satisfying the above constraints should have Gaussian shape. We use the following discrete approximations of Gaussian functions to serve as generating kernels with subsampling factor two, three, and five

$$
\begin{aligned}
K_{2} & =\frac{1}{16}\left(\begin{array}{lllllll}
1 & 4 & 6 & 4 & 1
\end{array}\right) \\
K_{3} & =\frac{1}{264}\left(\begin{array}{lllllll}
3 & 22 & 66 & 82 & 66 & 22 & 3
\end{array}\right) \\
K_{5} & =\frac{1}{5120}\left(\begin{array}{lllllll}
1 & 74 & 299 & 725 & 950 & 1022 & 950 \\
725 & 299 & 74 & 1
\end{array}\right) .
\end{aligned}
$$

\section{EXPERIMENTS}

\section{A. Synthetic Junction Examples}

In this section we illustrate some examples of junction characterization using the approximate steerability and its hierarchical version. In Figs. 4 and 5, synthetic line junctions and edge junctions are shown. The corresponding signatures $S(\theta)$ and $D S(\theta)$ characterize them correctly. The small deviations in Fig. 5 come from the fact that an edge can only be represented by two pixels in the grid, while we cannot set the center of a wedge between two pixels.

The robustness of our method against noise is shown in Fig. 6. The edge junction is disturbed with increasing random noise. Even in the very noisy case the junction is well characterized. The keypoints in Fig. 7 are deviated from the central positions of the masks. Though the signatures have some variations, we can still characterize the junctions.

In Fig. 8, we compare the performance of both steerability approaches. A complex junction called "Siemens star" with 16 edges spans the orientation space uniformly. Applying the steerable wedge filter we even have to use 90 basis filters to achieve the same orientation resolution as applying the Gaussian averaging steerable filter. Hence, we need about eleven times as many multiplications and thirteen times as many additions using the steerable wedge filter as using the Gaussian averaging steerable filter. This demonstrates the advantages of the local decomposition scheme in the approximate steerability.

Fig. 9 is an example to solve the orientation scale problem applying a polar pyramid. The junction is composed of two blurred edges, a wide line and two close lines. We use a polar pyramid with four levels to characterize it. With the increase of the pyramid level the orientation of the wide line and blurred edges are characterized more and more distinctly. However, in the mean time the responses of two close lines seem more and more like one line. This is exactly the demonstration of orientation scale problem.

\section{B. Real Experiments}

We have observed the successful behavior of the approximate steerability on synthetic junction characterizations. Here we show some real experimental results. One example is the parkbench picture used in [23] (Fig. 10). In comparison to the steerable wedge filter [23] with 30 basis filters our method characterizes the directions of junctions more distinctively. This is explicitly presented by the " $T$ " junction in $\mathrm{D}$ where the blurred edge near $180^{\circ}$ is better characterized with the approximately steerable filter. We see also that our approach is relatively more sensitive to high frequency components due to the differentiation.

In Fig. 11, we show the high orientation resolution of the approximate steerability. While the steerable wedge filter [23] with 90 basis filters only detects the dominant dark line between the lips of Lena, the approximately steerable filter characterizes edges of two lips distinctly. This may be very useful in facial expression analysis.

Another real example is presented in Fig. 12. The kernel centers are displaced from the keypoints of the junctions. The results show that both steerable wedge filter and approximately steerable filter are stable with respect to the offsets of keypoints while the Gaussian approach achieves higher orientation resolution with lower cost.

A real example with varying scales is further presented in Fig. 13. A child's left eye corner can be regarded as a combination of irregular wide lines and blurred edges disturbed by noise. The characterizing results from different pyramid levels form a complete set of signatures providing information at different orientation scales.

\section{CONCLUSION}

Most current orientation steerability approaches are based on the Fourier decomposition of the steered filter with respect to orientation. Although they are optimal with respect to approximation error, they suffer from the uncertainty principle: Due to the sampling of the spectral domain with Dirac functions the filters have wide spatial supports. In order to achieve high orientational resolution a huge number of basis filters must be applied.

We proposed a new approach to obtain the orientation signature for junction characterization. We called it approximate steerability because it enables the approximation of a continuous response with respect to orientation based on a number of basis filter responses. These filters are designed in the spatial domain so that they have narrow support. We used 0th- and firstorder derivatives of Gaussians to characterize lines and edges, respectively. Unfortunately, the 0th- and first-order derivative cannot be combined into a quadrature pair with the 0th as even and the 1st derivatives as odd part, respectively. Such a pair produces a significant energy in the negative frequencies and does not provide a phase independent magnitude response. However, in most tasks in vision lines and edges have totally different meaning. Therefore, a phase-independent magnitude response is not so helpful without the phase. Instead, our approach provides two signatures ( 0 th and 1st) characterizing the closeness to lines and edges, respectively. 
Last we mention the narrow support of the basis functions of our approach enables a high orientational resolution with a moderate computational load. We showed this difference to earlier approaches both in theory as well as in real images of junctions.

\section{ACKNOWLEDGMENT}

The authors would like to thank H. Farid, G. Birkelbach, H. Scharr, M. Michaelis, T. Bülow, V. Krüger, U. Heute, M. Felsberg and $\mathrm{H}$. Chen for their helpful suggestions and discussions. H. Farid has kindly provided the software for the steerable wedge filter and the "parkbench" picture. The authors highly appreciate the reviewers' valuable comments which helped them on clarifying and improving the paper.

\section{REFERENCES}

[1] J. Babaud, A. P. Witkin, M. Baudin, and R. O. Duda, "Uniqueness of the Gaussian kernel for scale-space filtering," IEEE Trans. Pattern Anal. Machine Intell., vol. 8, pp. 26-33, 1986.

[2] D. H. Ballard and L. E. Wixson, "Object recognition using steerable filters at multiple scales," in IEEE Workshop Qualitative Vision, 1993, pp. $2-10$.

[3] W. Beil, "Steerable filters and invariance theory," Pattern Recognit. Lett., vol. 15, pp. 453-460, 1994.

[4] R. N. Bracewell, The Fourier Transform and Its Applications. New York: McGraw-Hill, 1986.

[5] P. J. Burt and E. H. Adelson, "The Laplacian pyramid as a compact image code," IEEE Trans. Commun., vol. 31, pp. 532-540, 1983.

[6] P.-E. Danielsson, "Rotation-invariant linear operators with directional response," in Proc. Int. Conf. Pattern Recognition, Miami, FL, Dec. 1980.

[7] J. G. Daugman, "Uncertainty relation for resolution in space, spatial frequency and orientation optimized by two-dimensional visual cortical filters," J. Opt. Soc. Amer., vol. 2, no. 7, pp. 1160-1169, 1985.

[8] W. Förstner, "A framework for low level feature extraction," in Proc. 3rd Eur. Conf. Computer Vision, J. O. Eklundh, Ed. Stockholm, Sweden: Springer, May 2-6, 1994, vol. II, pp. 383-394.

[9] W. T. Freeman and E. H. Adelson, "The design and use of steerable filters," IEEE Trans. Pattern Anal. Machine Intell., vol. 13, pp. 891-906, 1991.

[10] H. Knutsson and G. H. Granlund, "Texture analysis using two-dimensional quadrature filters," in IEEE Comput. Soc. Workshop Comp. Architecture Patt. Anal. Image Database Mgmt., 1983, pp. 388-397.

[11] M. Michaelis, "Low level image processing using steerable filters," Ph.D. dissertation, Inst. Comput. Sci., University Kiel, Germany, 1995.

[12] M. Michaelis and G. Sommer, "Junction classification by multiple orientation detection," in Proc. 3rd Eur. Conf. Computer Vision, vol. I, Stockholm, Sweden, May 2-6, 1994, pp. 101-108.

[13] — , "Lie group approach to steerable filters," Pattern Recognit. Lett., vol. 16, pp. 1165-1174, 1995.

[14] V. S. Nalwa, A Guided Tour of Computer Vision. Reading, MA: Addison-Wesley, 1993.

[15] A. V. Oppenheim and R. W. Schafer, Digital Signal Processing. Englewood Cliffs, NJ: Prentice-Hall, 1975.

[16] L. Parida, D. Geiger, and R. Hummel, "Junctions: Detection, classification, and reconstruction," IEEE Trans. Pattern Anal. Machine Intell., vol. 20, no. 7, pp. 687-698, 1998.

[17] P. Perona, "Steerable-scalable kernels for edge detection and junction analysis," Image Vis. Comput., vol. 10, no. 10, pp. 663-672, 1992.
[18] — "Deformable kernels for earlyvision," IEEE Trans. Pattern Anal. Machine Intell., vol. 17, pp. 488-499, May 1995.

[19] T. Poggio and F. Girosi, "Networks for approximatation and learning," Proc. IEEE, vol. 78, pp. 1481-1497, Sept. 1990.

[20] B. Robbins and R. Owens, "2-D feature detection via local energy," Image Vis. Comput., vol. 15, pp. 353-368, 1997.

[21] K. Rohr, "Recognizing corners by fitting parametric models," Int. J. Comput. Vis., vol. 9, no. 3, pp. 213-230, 1992.

[22] - "On the precision in estimating the location of edges and corners," J. Math. Imag. Vis., vol. 7, pp. 7-22, 1997.

[23] E. P. Simoncelli and H. Farid, "Steerable wedge filters for local orientation analysis," IEEE Trans. Image Processing, vol. 5, pp. 1377-1382, Sept. 1996.

[24] E. P. Simoncelli, W. T. Freeman, E. H. Adelson, and D. J. Heeger, "Shiftable multi-scale transforms," IEEE Trans. Inform. Theory, vol. 38, no. 2, pp. 587-607, 1992.

[25] G. Sommer, M. Michaelis, and R. Herpers, "The SVD approach for steerable filter design," in IEEE Int. Symp. Circuits Systems, vol. V, Monterey, CA, May 31-June 3, 1998, pp. 349-353.

[26] P. C. Teo and Y. H. Or, "Lie generators for computing steerable functions," Pattern Recognit. Lett., vol. 19, no. 1, pp. 7-17, 1998.

Weichan Yu received the B.S. and M.S. degrees in electronic engineering from Tsinghua University and Wuhan Technical University of Surveying and Mapping, China, in 1993 and 1995, respectively. Since 1996 he has been a DAAD (German Academic Exchange Service) Scholarship holder pursuing the Ph.D. degree in the Cognitive Systems Group, Christian Albrechts University, Kiel, Germany.

His major research interests are signal processing, computer vision, and image analysis.

Kostas Daniilidis received the Diploma degree in electrical engineering from the National Technical University of Athens, Athens, Greece, in 1986 and the $\mathrm{Ph} . \mathrm{D}$. degree in computer science from the University of Karlsruhe, Karlsruhe, Germany, in 1992.

$\mathrm{He}$ is an Assistant Professor of computer and information science, University of Pennsylvania, Philadelphia, affiliated with the interdisciplinary GRASP Laboratory. Prior to this, he was with the Cognitive Systems Group, University of Kiel, Kiel, Germany. His research centers on omnidirectional vision and vision techniques for tele-immersion and augmented reality. He has served as Member of many Program Committes and he is a reviewer for multiple journals.

Dr. Daniilidis was the Chair of the IEEE Workshop on Omnidirectional Vision 2000 .

Gerald Sommer received the Diploma degree in physics and the Ph.D. degree in physics from Friedrich-Schiller-Universität Jena, Germany, in 1969 and 1975 , respectively, and the Habilitation degree in engineering from Technical University Ilmenau, Germany, in 1988.

From 1969 to 1991, he was with several departments of Friedrich-SchillerUniversity Jena. From 1991 to 1993, he was the Head of the Division for Medical Image Processing, Research Center for Environment and Health (GSF-Medis), Munich-Neuherberg, Germany. Since 1993, he has been Professor of computer science at the Christian Albrechts University Kiel, Kiel, Germany. He is leading the research group in cognitive systems. His main interests are the design of behavior-based systems. His research covers signal theory and signal processing, neural computation for pattern recognition, and robot control. 